University of Nebraska - Lincoln DigitalCommons@University of Nebraska - Lincoln

2007

\title{
Simulation of Richtmyer-Meshkov instability by sixth-order filter methods
}

Helen C. Yee

NASA Ames Research Center, yee@nas.nasa.gov

Bjorn Sjögreen

Royal Institute of Technology, bjorns@nada.kth.se

Follow this and additional works at: http://digitalcommons.unl.edu/nasapub

Yee, Helen C. and Sjögreen, Bjorn, "Simulation of Richtmyer-Meshkov instability by sixth-order filter methods" (2007). NASA Publications. 262.

http://digitalcommons.unl.edu/nasapub/262

This Article is brought to you for free and open access by the National Aeronautics and Space Administration at DigitalCommons@University of Nebraska - Lincoln. It has been accepted for inclusion in NASA Publications by an authorized administrator of DigitalCommons@University of Nebraska - Lincoln. 


\title{
Simulation of Richtmyer-Meshkov instability by sixth-order filter methods
}

\author{
H. C. Yee · B. Sjögreen
}

Received: 9 January 2007 / Accepted: 10 July 2007 / Published online: 1 September 2007 (C) US Government 2007

\begin{abstract}
Simulation of a 2-D Richtmyer-Meshkov instability (RMI), including inviscid, viscous and magnetic field effects was conducted comparing recently developed sixthorder filter schemes with various standard shock-capturing methods. The suppression of the inviscid gas dynamics RMI in the presence of a magnetic field was investigated by Samtaney and Wheatley et al. Numerical results illustrated here exhibit behavior similar to the work of Samtaney. Due to the different amounts and different types of numerical dissipation contained in each scheme, the structures and the growth of eddies for the chaotic-like inviscid gas dynamics RMI case are highly grid size and scheme dependent, even with many levels of refinement. The failure of grid refinement for all studied numerical methods extends to the viscous gas dynamics case for high Reynolds number. For lower Reynolds number, grid convergence has been achieved by all studied methods. To achieve similar resolution, standard shock-capturing methods require more grid points than filter schemes and yet the CPU times using the same grid for all studied methods are comparable.
\end{abstract}

Communicated by M. Onofri, Guest Editor, ISIS17.

This paper is based on work that was presented at the 17 th International Shock Interaction Symposium (ISIS17), Rome, Italy, 4-8 September 2006.

H. C. Yee ( $\varangle)$

NASA Ames Research Center, Moffett Field, CA, USA

e-mail: yee@nas.nasa.gov

B. Sjögreen

NADA, KTH, Stockholm, Sweden

e-mail: bjorns@nada.kth.se

\section{Numerical method and objective}

Efficient and accurate numerical simulation of complex multiscale fluid and plasma flows containing strong shocks, and high shear turbulence mixings is computationally very challenging due to the wide range of temporal and spatial length scales. Numerical methods designed to treat discontinuities and shocks are inherently dissipative for turbulence, and methods designed for turbulence are ineffective for discontinuities. Among other issues, capturing this type of interaction in an accurate and efficient manner requires novel algorithms and effective use of software tools which allow the full benefit of the new algorithms to be realized on the terascale and petascale supercomputer architectures.

Methods commonly used for multiscale problems containing shock waves rely on switching between spectral or high order compact schemes and shock-capturing schemes, and they are not practical for multiscale shock/turbulence interactions. Frequent switching between these two types of schemes can create severe numerical instability. The recently developed highly parallelizable class of high order filter schemes [18,28,29,31-34] focused primarily on overcoming the aforementioned stumbling blocks. This class of filter schemes does not rely on switching between schemes to avoid the related numerical instability. They have built-in flow sensors to control the amount and types of numerical dissipation only where needed, leaving the rest of the flow region free of numerical dissipation. Instead of solely relying on very high order high-resolution shock-capturing methods for accuracy, the filter schemes take advantage of high order spatial central schemes and the effectiveness of the non-linear dissipation contained in good shock-capturing schemes and standard linear high order filters as stabilizing mechanisms at locations where needed. 
The four main features of this class of high order schemes are: (a) multiresolution wavelet decomposition of the computed flow data as sensors for adaptive numerical dissipative control, (b) multistep filter to accommodate efficient application of different numerical dissipation models and different spatial high order base schemes, (c) a unique idea in solving the ideal conservative MHD system (a non-strictly hyperbolic conservation law) without having to deal with an incomplete eigensystem set while at the same time ensuring that correct shock speeds and locations are computed, and (d) minimization of the divergence of the magnetic field numerical error. The method consists of two steps, a full time step of spatially high order non-dissipative (or very low dissipative) base scheme and an adaptive multistep filter consisting of the products of wavelet based flow sensors and linear and non-linear numerical dissipations to filter the solution. The adaptive numerical dissipation control idea is very general and can be used in conjunction with spectral [3], spectral element [2], finite element, discontinuous Galerkin [15], finite volume and finite difference spatial base schemes. The type of shock-capturing scheme used as non-linear dissipation is very general and can be any dissipative portion of a high resolution TVD, MUSCL, ENO, or weighted ENO (WENO) shock-capturing method [7,15,27]. By design, the flow sensors, different choice of high order base schemes and numerical dissipation models are stand-alone modules. A whole class of low dissipative high order schemes can be derived at ease, making the resulting computer software very flexible and widely applicable. See [18,21-24,28-34] for the development of the multiscale and multiphysics high order filter schemes. See $[6,9,10,14,20]$ and references cited therein for multi-resolution analysis of grid functions.

During the last 5 years a highly parallel 3-D NavierStokes/MHD computer code using the MPI library was built that contains our filter schemes and six standard shock-capturing schemes. This code has been well tested and debugged. The proof of concept includes:

- Numerical experiments on over three dozen representative test cases for inviscid and viscous 1-D, 2-D gas dynamics problems as well as ideal and non-ideal MHD test cases. These test cases range from simple 1-D shock tube problems to multiscale and multiphysics problems. The majority of the test cases are either with exact, known converged solutions, or by 5-6 levels of grid refinement of known methods as reference solutions. Stability and accuracy of our filter schemes were then assessed according to these findings.

- Comparison among the second-, fourth-, sixth- and eighth-order central base schemes.

- Comparison among different filter approaches with standard TVD, MUSCL and fifth-order WENO (WENO5) schemes.
- Comparison among the multistep filters and single step filters (see [35] for the definition and procedures).

- Proper numerical boundary condition treatment for high order base schemes are taken into consideration. Comparison among sixth-order central spatial base schemes $[34,35]$ with two different summation-by-parts (SBP) numerical boundary operators $[12,13,30]$ with the sixthorder compact spatial base scheme.

Studies show that our adaptive numerical dissipation control can accurately simulate a wide spectrum of flow speeds, flow types and governing equation sets, namely, from nearly incompressible to high speed shock/turbulence/combustion multiscale gas dynamics and MHD plasma flows. The filter scheme is more accurate and efficient than the standard structured or unstructured method commonly used in gas dynamics and plasma applications. In many instances, grid convergence was achieved by our high order filter schemes but not by standard second-order shock-capturing methods using the same grid sequence. The objective of this work is to further investigate the performance of our sixth-order low dissipation filter schemes employing the sixth-order compact and non-compact central base schemes [34-36] to simulate a 2-D Richtmyer-Meshkov instability (RMI), including inviscid, viscous and magnetic field effects. This is a sequel to the study initiated in [25].

For generality of discussion, we denote the standard spectral filter [3], compact filter [4] and non-compact high order linear numerical dissipation as high order linear numerical dissipations (or linear filter). In contrast, we denote the dissipative portion of any high resolution shock-capturing scheme as non-linear numerical dissipation since these dissipations are non-linear even if one applies the scheme to a linear conservation law. When non-linear dissipations are applied in a filter approach, we denote the approach as non-linear filters. Although non-linear numerical dissipations can suppress spurious high frequency oscillations, they might not be as effective as the standard high order linear dissipations (or linear filters). With the appropriate wavelet flow sensors, locations of spurious high frequency oscillations, locations of shocks and high gradient regions, and locations of large vortices or vortex sheets can be detected separately. The appropriate numerical dissipations are then applied to these locations leaving the remaining regions free of added numerical dissipation.

For the numerical results shown below, except for WENO, the van Leer version of the van Albada limiter is used in the non-linear filter step. For the second-order MUSCL scheme, the limiter is applied to the primitive variables. For the rest of the studied methods, the limiter is applied to the local 1-D characteristic variables [30]. All methods employed the Roe's approximate Riemann solver for the gas dynamics case 
and the Gallice approximate Riemann solver [5] for the MHD case using our method of solving the conservative MHD system [32]. See [35,36] and references cited therein for the recent progress of the filter schemes.

\section{RMI test problem}

RMI occurs when an incident shock accelerates an interface between two fluids of different densities. This interfacial instability was theoretically predicted by Richtmyer [16] and experimentally observed by Meshkov [11]. Numerical studies were performed for two RMI problems, namely, the RMI problem discussed in [1] and the RMI problem discussed in [17]. The first RMI problem is less interesting and the accuracy and efficiency of our filter schemes is similar to the second RMI problem. For the present study, the RMI problem investigated by Samtaney [17] and Wheatley et al. [26] as indicated in Fig. 1 has been chosen for detailed discussion. The mathematical models are the 2-D Euler gas dynamics equations and the ideal MHD equations. The computational domain is $-2<x<6$ and $0<y<1$. A planar shock at $x=-0.2$ is moving (left to right) toward

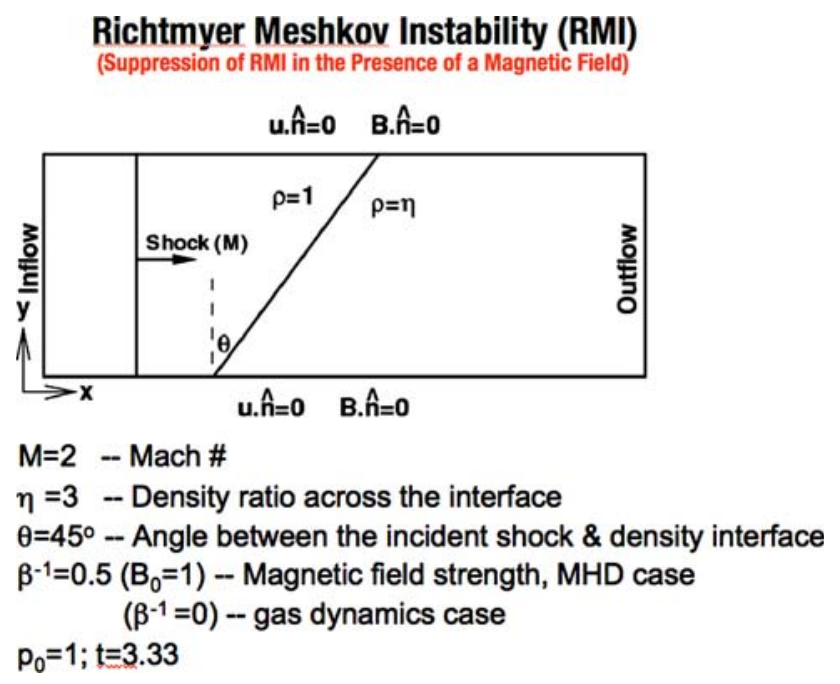

Fig. 1 Problem definition

Fig. 2 Time evolution by CEN66+HYfi using a $6,401 \times 801$ grid an incline density interface of angle $\theta$ with the lower end initialized at $x=0$. The density ratio across the interface is denoted by $\eta$, and the non-dimensional strength of the magnetic field $\beta=2 p_{0} / B_{0}^{2}$, where the initial pressure in the preshocked regions is $p_{0}=1$, and $B_{0}$ is the initial magnetic field. The initial magnetic field is uniform in the $(x, y)$ plane and perpendicular to the incident shock front. Numerical results shown below are for $M=2, \theta=45^{\circ}, \eta=3$, $\beta^{-1}=0$ (Euler gas dynamics) and $\beta^{-1}=0.5$ (magnetic field present). The computation stops at an evolution time $t=3.33$. For this set of parameters and all studied numerical schemes, RMI occurs for the inviscid gas dynamics case but not for the MHD case for the entire time evolution. Our Euler numerical results exhibit behavior similar to the study of Samtaney. Figure 2 shows density contours of six snapshots of the time evolution of the Euler gas dynamics simulation by our sixth-order filter scheme CEN66+HYfi using a 6,401 $\times 801$ grid. Here CEN66+HYfi refer to the use of the sixth-order central base scheme and the dissipative portion of Harten-Yee scheme [27] in conjunction with wavelets flow sensor as the non-linear filter. The fine scale eddy structures can only be captured by low dissipative high order methods or by adaptive grid refinements. See subsequent figures for some illustration. In addition to the inviscid gas dynamics case, the present study also investigate the effect of physical viscosity on the RMI that was not considered in Samtaney.

\section{Numerical results}

Six aspects of the numerical study were considered, namely, (a) suppression of the RMI in the presence of a magnetic field, (b) comparison between compact and non-compact central base schemes, (c) comparison among three non-linear filters with three standard shock-capturing methods, (d) effects of linear dissipation and non-linear filter on eddy structures, (e) effects of physical viscosity on the eddy structures, and (f) grid refinement study for the $R e=10^{4}, 10^{5}$ cases. The discussion is broken into four subsections. Section 3.1 discusses aspects (a) and (b). Section 3.4 discusses aspects (e) and (f).

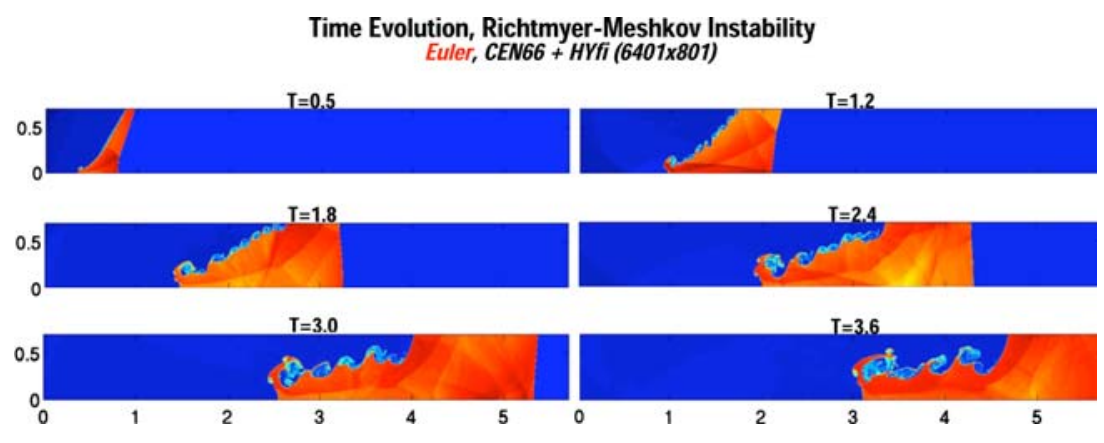


Fig. 3 Comparison between Euler gas dynamics and ideal MHD for the sixth-order compact spatial base scheme (left) and the sixth-order central (non-compact) spatial base scheme (right) using a $(801 \times 101)$ grid at $t=3.33$. MHD solutions shown are mirror images of the original computations

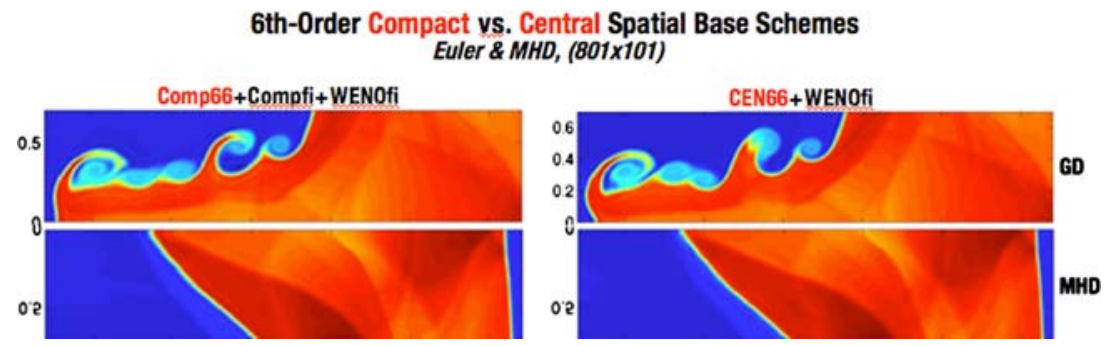

3.1 Suppression of the RMI in the presence of a magnetic field and comparison between compact and non-compact base schemes

Computations by the sixth-order centered spatial compact base scheme [8] with the compact linear filter [4], in conjunction with a second step non-linear WENO5 filter (WENOfi) denoted by Comp66+Compfi+WENOfi using a $801 \times 101$ grid is shown in Fig. 3 (left) for the inviscid gas dynamics (GD) and the ideal MHD equations. See [35,36] for a discussion of multistep linear and non-linear filters. Here WENOfi means the dissipative portion of the fifth-order WENO scheme (WENO5) in conjunction with our wavelet flow sensor as the non-linear filter [33-35]. The same computation using the sixth-order central spatial (non-compact) base scheme in conjunction with WENOfi denoted by CEN66+ WENOfi is shown in Fig. 3 (right). The classical fourthorder Runge-Kutta temporal discretization (RK4) is used for the sixth-order compact and non-compact filter schemes. For the MHD case, both solutions have been converged when compared with the reference solution by CEN66+WENOfi and CEN66+HYfi using a 6,401 $\times 801$ grid. For both base schemes, suppression of the RMI in the presence of the magnetic field was observed for the MHD cases. Grid convergence was achieved by both methods with only two levels of grid refinement for this MHD case.

For the gas dynamics case, however, their resulting solutions are different and it is difficult to judge the accuracy among methods. To show their behavior, the same comparison of gas dynamics computations using a 1,601 $\times 201$ grid was conducted and shown in [36]. Their computed Eddy structures are different. Thus a finer grid refinement is needed for the gas dynamics case to evaluate the situation. See the next section for an investigation.

Computations using Comp66+WENOfi (i.e., without the linear compact filter step) or Comp66+Compfi (i.e., without the non-linear WENOfi filter step) indicate spurious oscillations around shock regions. The present study arrives at the same conclusion drawn in $[28,34]$ on the behavior of compact spatial schemes for problems containing multiscale shock interaction. High order compact schemes are methods of choice for many incompressible and low speed turbulent/acoustic flows due to their advantage of requiring very low number of grid points per wavelength. In the presence of multiscale shock interactions, however, this desired property of high order compact base schemes seems to have diminished in both the gas dynamic and MHD test cases that we have studied (compared with the same order of accuracy of non-compact central base schemes). Also the compact spatial base scheme requires more CPU time per time step and it is less compatible with parallel computations than the central spatial base scheme. Consequently, the compact spatial base scheme requires added $\mathrm{CPU}$ time in a parallel computer framework.

\subsection{Comparison among three sixth-order filters for the Euler RMI}

As mentioned earlier, the type of shock-capturing scheme used as the non-linear dissipative portion of the non-linear filter is very general and can be any dissipative portion of a high resolution TVD, MUSCL, ENO, or WENO shockcapturing method. Here the dissipative portion of three shockcapturing schemes as part of the non-linear filters are considered. The base scheme used in this case is the sixthorder non-compact central scheme using wavelet as the flow sensor and RK4 as the temporal discretization (CEN66). Figure 4 shows the inviscid gas dynamics grid refinement comparison among a second-order MUSCL, CEN66+MUSfi (sixth-order filter using the dissipative portion of MUSCL as part of the non-linear filter), and CEN66+WENOfi for four grids $(801 \times 101,1,601 \times 201,3,201 \times 401,6,401 \times$ $801)$. Here, computations using a second-order MUSCL and a second-order Runge-Kutta temporal discretization (RK2) is denoted by MUSCL. Not shown is the same computation using CEN66+HYfi (sixth-order filter using the dissipative portion of Harten-Yee TVD scheme as part of the non-linear filter). For similar resolution, the standard shockcapturing scheme MUSCL requires nearly three times finer grid size per spatial direction than CEN66+MUSfi, CEN66+HYfi and CEN66+WENOfi. The difference in the computed global structures and eddy structures among methods is not pronounced for the $801 \times 101$ grid. As the grid is 


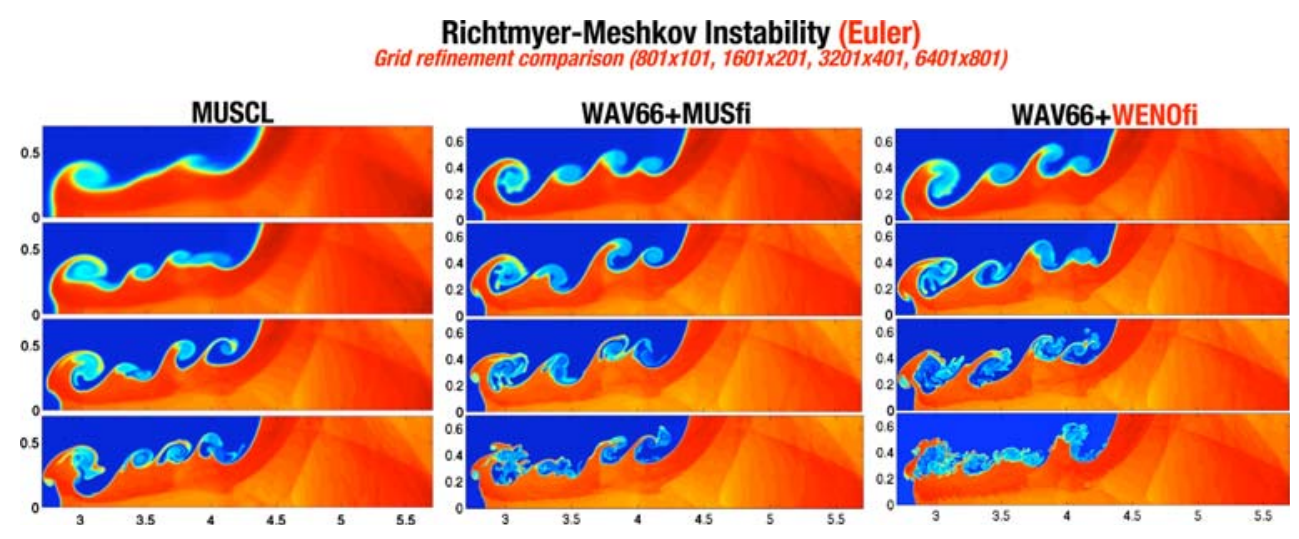

Fig. 4 Euler RMI: Grid refinement study of the second-order MUSCL (WAV66+MUfi, left), CEN66+MUSfi (WAV66+WENOfi, middle) and CEN66+WENOfi $($ right $)$ at $t=3.33$ using $(801 \times 101),(1,601 \times 201),(3,201 \times 401)$ and $(6,401 \times 801)$ grids

refined, the computed global structures as well as the eddy structures are different among the three filter methods and they are very different from the Samtaney adaptive mesh refinement (AMR) simulation with an equivalent uniform grid of $16,384 \times 2,048$. The fine scale structure of the eddies are not captured by the three standard shock-capturing methods, Harten-Yee (with RK2), MUSCL and WENO5 (with RK4) using the same grid sequence. In addition, the resulting eddy structures are also very different from each other. It appears that grid refinement cannot be achieved among the studied methods.

We use the expression "achieving grid convergence" or "a mesh resolved solution" of a numerical method for a chosen mathematical model to mean that the computed solution converges to a discrete solution having the same global structure as well as same key fine scale structures when compared with well-tested commonly used schemes under grid refinement. The chosen model is assumed to have no known solution. We also use the term "failure of grid refinement" to mean:

"For a chosen model, one cannot obtain a grid convergence solution by well tested commonly used numerical schemes with fine grid refinement. Their solution structures are different from method to method and from one grid to another and yet each scheme does not diverge during the entire time evolutionary process and grid sequence process. Aside from having very different fine scale structures, the global structures by each method do not indicate a trend of convergence to the same global feature as the grid is refined."

Due to the fact that the global structures of the Euler gas dynamics case change from method to method and from grid size to grid size on the considered fine grid sequence, the different behavior of all studied methods prompted us to investigate the effect of different numerical dissipation coefficients contained in the filter schemes on the eddy structures of the simulated flows. The next subsection discusses the effects of high order linear dissipation and non-linear filters on eddy structures of the Euler RMI.
3.3 Effects of linear dissipation and non-linear filters on eddy structures of the Euler RMI

For strong shock interactions and/or steep gradient flows, a small amount of high order linear dissipation can be added to the base scheme step to reduce the time step constraint and to improve numerical stability. For example, an eighth-order linear dissipation with the sixth-order centered non-compact and compact base schemes to approximate an inviscid flux derivative, e.g., $F(U)_{x}$, where $U$ is the vector of conservative variables is written as

$$
\begin{aligned}
& \frac{\partial F}{\partial x} \approx D_{06} F_{j}+d(\Delta x)^{7}\left(D_{+} D_{-}\right)^{4} U_{j}, \\
& \frac{\partial F}{\partial x} \approx C_{06} F_{j}+d(\Delta x)^{7}\left(D_{+} D_{-}\right)^{4} U_{j},
\end{aligned}
$$

where $D_{06}$ is the standard sixth-order accurate centered difference operator, and $D_{+} D_{-}$is the standard second-order accurate centered approximation of the second derivative. Here $F_{j}$ and $U_{j}$ denote $F(U)$ and $U$ at grid point $j$. The small parameter $d$ is a scaled value (e.g., spectral radius of $\left.\frac{\partial F}{\partial U}\right)$ in the range of $0.00001-0.0005$, depending on the flow problem, and has the sign which gives dissipation in the forward time direction. The second terms in (1) and (2) denoted by AD8 (for notation purpose) are the eighth-order linear dissipation added to the sixth-order base scheme with $d$ the coefficient of AD8. The $D_{06}$ operator is modified at boundaries in a stable way by the so called summation-by-part (SBP) operators $[12,13,30]$. The linear numerical dissipation operator $D_{+} D_{-}$is modified at the boundaries to be semi-bounded [19]. The symbol $C_{06}$ in (2) denotes the sixth-order centered compact operator. Some comparison of the two base schemes has been reported in [34]. Previous studies [32,34] indicated that the two base schemes might require different amounts of linear dissipation (or linear filter) and non-linear filter, depending on the test problem. An earlier section shows the behavior of single step and multistep filters by the compact 
Fig. 5 Euler RMI: Effect of linear dissipation (AD8) and non-linear filter by two different filters and AD8 coefficients of $(0,0.0005,0.001,0.002)$ using a $(6,401 \times 801)$ grid

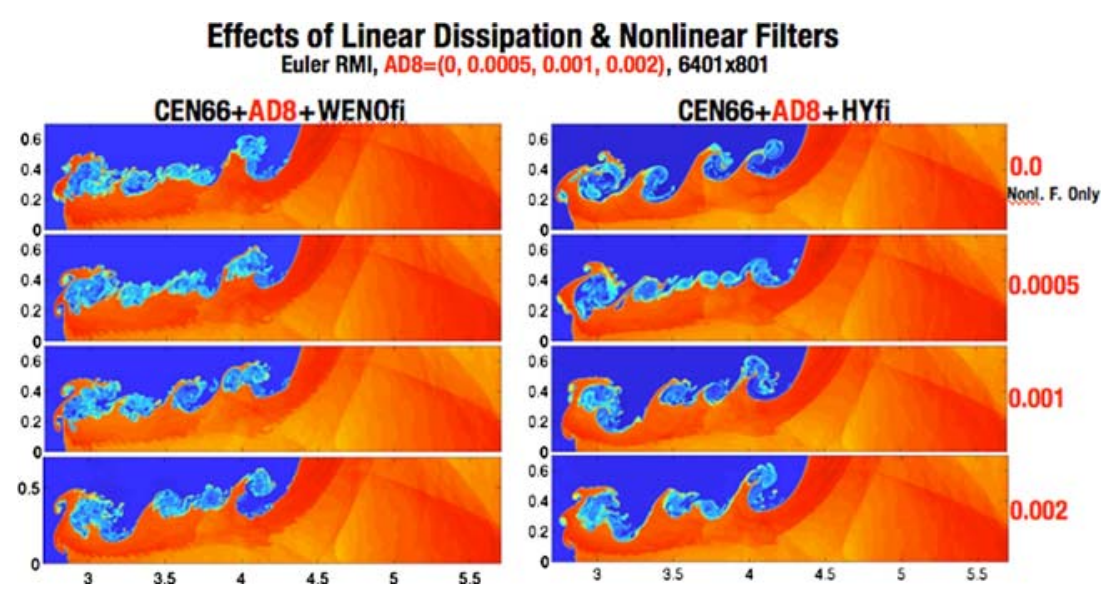

base scheme for the RMI problem. This section illustrates yet another aspect of the different combinations of numerical dissipation for the present chaotic-like RMI flows. Aside from improving numerical stability due to long time integration related spurious high frequency oscillations, the inclusion of the non-zero AD8 can have a different affect on the locations where non-linear filters are utilized than without AD8. For example, for the coefficient $d=0$ of AD8, the wavelet sensor would indicate that non-linear filters are needed at locations of spurious high frequency oscillations as well as at discontinuity locations that experience Gibbs phenomena. However, for AD8 with $d \neq 0$, the linear filter would damp out some or all of the high frequency oscillation locations. However, in the actual case, this is a very dynamic procedure and highly problem dependent, and base scheme and filter term dependent, especially when one is dealing with a chaotic-like flow. Ideally, AD8 should contain a proper flow sensor to indicate where linear dissipation is needed. For the MHD system, in order to preserve the divergence-free property of the base scheme step on Cartesian grid, it is more desirable to apply a small amount of AD8 uniformly. It is noted that this combination of linear dissipation and nonlinear filter might not be the optimal approach in general. The following gives just one simple-minded aspect of this study.

The effect of high order linear dissipation added uniformly to the non-compact central base scheme in conjunction with non-linear filters for the RMI is discussed next. Figure 5 shows the effect of linear dissipation AD8 added to the base scheme in conjunction with two different filters (CEN66+AD8+WENOfi and CEN66+AD8+HYfi ) for three linear dissipation coefficients of $\mathrm{AD} 8$ with $d=0.0005$, $0.001,0.002$ in $(1)$ using a $(6,401 \times 801)$ grid. The top subfigures show the computations using only a non-linear filter $(\mathrm{AD} 8=0)$. The rest of the sub-figures are computations using three different non-zero AD8 coefficients. With such a fine grid, the eddy structures are very different. Traditionally, when dealing with non-chaotic turbulent type flows, grid refinement can serve as a measure of the accuracy and convergence property of the numerical methods. (In this case, the grid refinement is based on the comparison of different AD8 coefficients using the same non-linear filter as well as comparison with three different non-linear filters.) However, due to the chaotic-like nature of the present Euler RMI, the small amount of high order linear dissipation present on the spatial base scheme actually alters the type of governing equation that we are solving. In effect, we are solving the Navier-Stokes equations with a linear viscosity term. This in conjunction with the adaptive non-linear filter (i.e., shockcapturing dissipations were employed at locations that are dictated by the wavelet flow sensor) results in a complex interplay of different types and amount of numerical dissipation which can alter the chaotic pattern of the flow. The study can serve as a good example of failure of grid refinement for unsteady chaotic-like inviscid flow. As the grid is refined (in conjunction with different amounts and types of numerical dissipations contained in each scheme), smaller and smaller eddies are formed which combine to affect global flow through the energy cascade effect.

Note that for the inviscid gas dynamics RMI computations, the three filter schemes using the first three grids require similar CPU times for the entire time evolution for each grid. However, for the finest grid, CEN66+WENOfi requires nearly twice the CPU time than that of CEN66+HYfi and CEN66+MUSfi. In general, CEN66+MUSfi is more dissipative than the other two filters. Part of the reasons is that the non-linear dissipative portion of MUSCL is more diffusive than Harten-Yee and WENO5.

\subsection{Effect of physical viscosity on the RMI}

The failure of grid refinement of the Euler RMI by all studied methods prompted us to investigate the same RMI in the presence of physical viscosity. Our aim is to obtain a rough estimate of the Reynolds number if the viscous RMI ceases to exist. For Navier-Stokes computations with Reynolds 
Fig. 6 Viscous RMI: Effect of Reynolds numbers (top to bottom: Euler, $\left.\operatorname{Re}=10^{5}, 10^{4}, 10^{3}\right)$ by two different filters using a $(1,601 \times 201)$ grid, CEN66+WENOfi (left) and CEN66+HYfi (right)

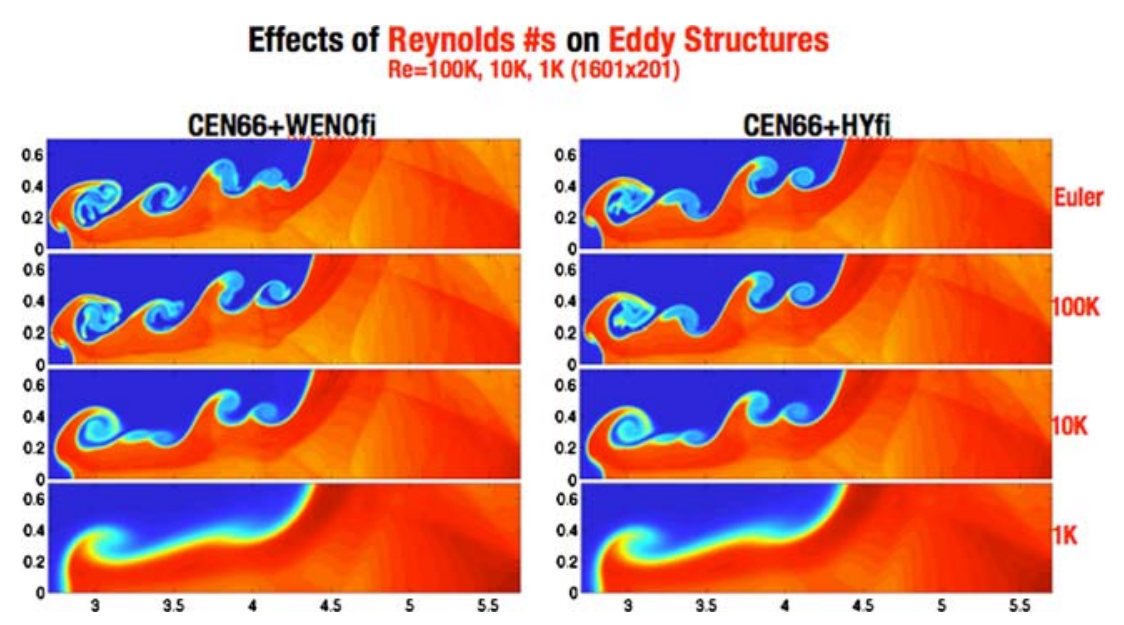

Viscous $\mathrm{RMI}, \mathrm{Re}=100,000$

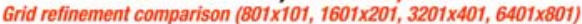
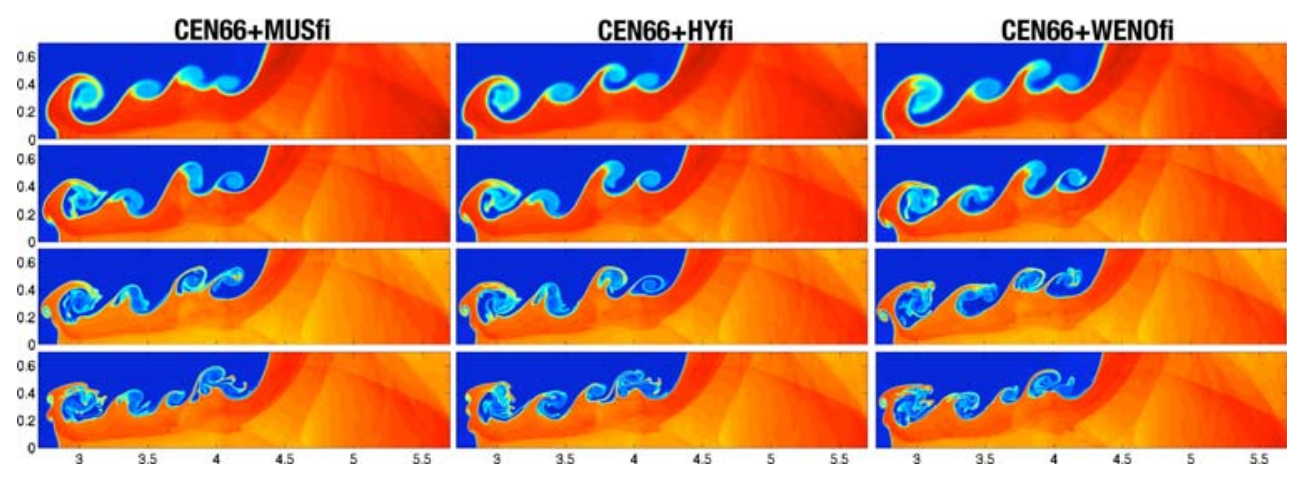

Fig. 7 Viscous RMI: Grid refinement study for $R e=10^{5}$ by CEN66+MUSfi (left), CEN66+HYfi (middle) and CEN66+WENOfi (right) using $(801 \times 101),(1,601 \times 201),(3,201 \times 401)$, and $(6,401 \times 801)$ grids

numbers higher than $10^{4}$, the same failure of grid refinement was encountered by all studied methods using the same grid sequence. In the presence of physical viscosity and for Reynolds number below $10^{4}$, grid refinement has been achieved by all studied methods. Figure 6 shows the computations of Euler and three different Reynolds numbers by CEN66+ WENOfi and CEN66+HYfi using a 1,601 × 201 grid. Judging from the 1,601 $\times 201$ grid shown in Fig. 6, it seems that all the computed Eddy structures for the studied Reynolds numbers are similar among schemes. From lessons learned on the Euler gas dynamics studies, a grid refinement study was performed for $R e=10^{5}$ and $R e=10^{4}$. Figures 7 and 8 show the grid refinement study for the two Reynolds numbers. Although the difference among the computed global structures and Eddy structures of the three filters is not as pronounced as their Euler counterparts, their Eddy structures are different from method to method and from one grid to another for the $R e=10^{5}$ case. As opposed to higher Reynolds number, for the same grid sequence with the finest grid, grid convergence was almost achieved for $R e=10^{4}$ except with a very slightly different eddy shape. It is anticipated that with the next level of grid refinement (i.e., for a
$12,800 \times 1,600$ grid) or by AMR, grid convergence would have been achieved for $R e=10^{4}$. Due to the high CPU time cost on this level of fine grid, no further investigation is planned.

For the high Reynolds number case, to achieve similar resolution, second-order MUSCL, second-order Harten-Yee and WENO5 required more than double the grid points in each spatial direction than that of filter schemes and yet the CPU time per grid point and time step using the same grid for most of the studied methods is comparable. Future work, including the bracketing of the bifurcation Reynolds number for the Navier-Stokes system where the Richtmyer-Meshkov instability ceases to exist is planned.

\section{Concluding remarks}

The efficiency and flexibility of the present class of low dissipative high order filter schemes are illustrated by the RMI test case. The efficiency rests on the fact that even though the multistep filter can be applied after each stage of the Runge-Kutta method or after a full time step of the Runge-Kutta method, 
Fig. 8 Viscous RMI: Grid refinement study for $R e=10^{4}$ by CEN66+WENOfi (left) and CEN66+HYfi (right) using $(801 \times 101),(1,601 \times 201)$, $(3,201 \times 401)$, and $(6,401 \times 801)$ grids

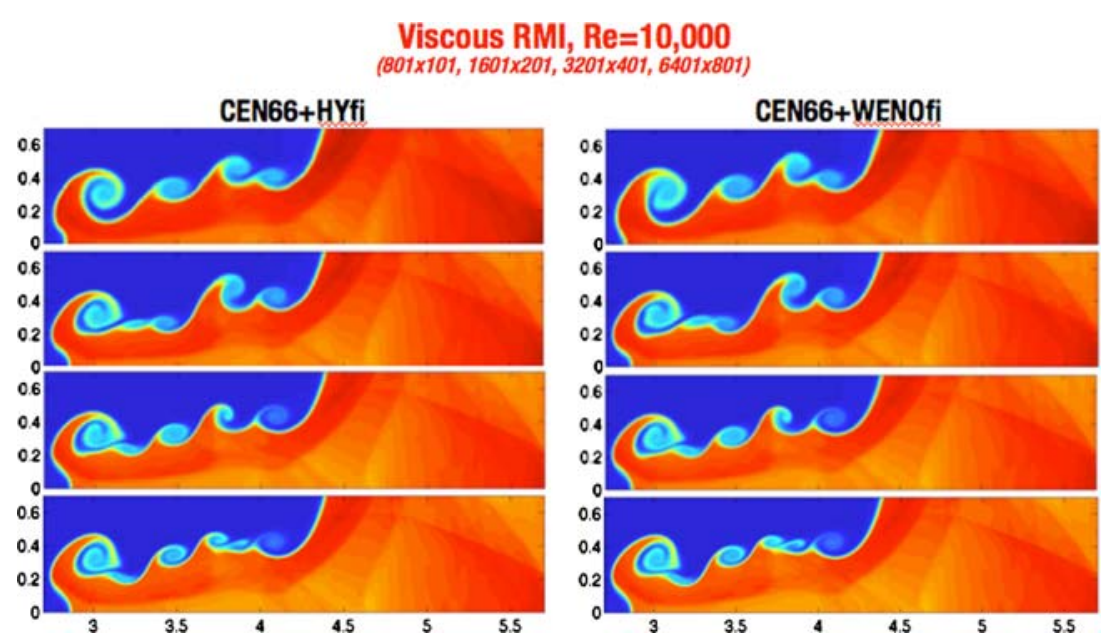

our present numerical studies and previous studies on many representative problems indicate that there is no difference in accuracy or stability among the two filter procedures. The latter procedure is very efficient as illustrated by the present simulation. The major CPU time intensive part of the computation is the non-linear filter. In fact, the latter sixth-order filter procedure using CEN66, in general, requires slightly more CPU time (20\%) per time step and grid point using the same grid than the Harten-Yee and MUSCL schemes. This is due to the fact that all three filter schemes require only one Riemann solver per time step per direction (independent of the time discretizations of the base scheme step) as opposed to two Riemann solves per time step per direction by the MUSCL and Harten-Yee schemes using a secondorder Runge-Kutta method. WENO5-RK4 requires at least twice the CPU time of all other methods since four Riemann solves per time step per direction are required by WENO5RK4. Another gain in efficiency is that CEN66+HYfi and CEN66+WENOfi exhibit similar accuracy for the RMI and all of the considered test cases studied previously. In other words, the dissipative portion of second-order shock-capturing schemes in conjunction with the wavelet sensor as nonlinear filters is sufficient. Consequently, the complication of dealing with a high order non-linear filter with wider grid stencils and higher order numerical boundary treatment can be avoided.

\section{References}

1. Abarzhi, S.I., Hermann, M.: New type of the interface evolution in the Richtmyer-Meshkov instability. Center for Turbulence Research Annual Research Briefs, pp. 173-183 (2003)

2. Fischer, P.F., Ho, L.-W., Karniadakis, G.E., Rønquist, E.M., Patera, A.T.: Recent advances in parallel spectral element simulation of unsteady incompressible flows. Comput. Struct. 30, 217-231 (1988)

3. Gottlieb, D., Hussaini, M.Y., Orszag, S.A.: Theory and applications of spectral methods, spectral methods for partial differential equations. In: Voigt, R., Gottlieb, D., Hussaini, M.Y. (eds.) SIAM, Philadelphia, pp. 1-54 (1984)

4. Gaitonde, D.V., Visbal, M.R.: Further development of a NavierStokes solution procedure based on higher-order formulas. AIAA Paper 99-0557 (1999)

5. Gallice, G.: Systéme D’Euler-Poisson, Magnétohydrodynamique et Schemas de Roe. Ph.D. Thesis, L'Université Bordeaux I (1997)

6. Harten, A.: Multiresolution algorithms for the numerical solution of hyperbolic conservation laws. Comm. Pure Appl. Math. 48, 1305-1342 (1995)

7. Jiang, G.-S., Shu, C.-W.: Efficient implementation of weighted ENO schemes. J. Comput. Phys. 126, 202 (1996)

8. Lele, S.K.: Compact finite difference schemes with spectral-like resolution. J. Comput. Phys. 103, 16-42 (1992)

9. Mallat, S.G.: A Wavelet Tour of Signal Processing, 2nd edn. Academic, San Diego (1999)

10. Mallat, S.G., Zhong, S.: Characterization of signals from multiscale edges. IEEE Trans. Pattern Anal. Mach. Intell. 14, 710-732 (1992)

11. Meshkov, Y.Y.: Instability of a shock wave accelerated interface between two gases. NASA Tech. Trans. NASA TT F-13074 (1970)

12. Nordstrom, J., Carpenter, M.H.: Boundary and interface conditions for high-order finite-difference schemes applied to the euler and Navier-Stokes equations. J. Comput. Phys. 148, 621-645 (1999)

13. Olsson, P.: Summation by Parts, Projections and Stability. I. Math. Comp. 64, 1035-1065 (1995)

14. Perrier, V., Philipovitch, T., Basdevant, C.: Wavelet Spectra Compared to Fourier Spectra. Publication of ENS, Paris (1999)

15. Qiu, J., Khoo, B.C., Shu, C.-W.: A numerical study for the performance of the Runge-Kutta discontinuous galerkin method based on different numerical fluxes. J. Comput. Phys. 212, 540-565 (2006)

16. Richtmyer, R.D.: Taylor instability in shock acceleration of compressible fluids. Commun. Pure Appl. Math. 13, 297 (1960)

17. Samtaney, R.: Suppression of the Richtmyer-Meshkov instability in the presence of a magnetic field. Phys. Fluids 15, L53-L56 (2003)

18. Sandham, N.D., Li, Q., Yee, H.C.: Entropy splitting for highorder numerical simulation of compressible turbulence. J. Comp. Phys. 178, 307-322 (2002)

19. Sjögreen, B.: High order centered difference methods for the compressible Navier-Stokes equations. J. Comput. Phys. 117, 67-78 (1995)

20. Sjögreen, B.: Numerical experiments with the multiresolution scheme for the compressible Euler equations. J. Comput. Phys. 117, 251 (1995) 
21. Sjögreen, B., Yee, H.C.: Multiresolution wavelet based adaptive numerical dissipation control for shock-turbulence computation. RIACS Technical Report TR01.01, NASA Ames Research Center, Oct 2000, J. Scient. Comput. 20, 211-255 (2004)

22. Sjögreen, B., Yee, H.C.: Grid convergence of high order methods for multiscale complex unsteady viscous compressible flows. J. Comput. Phys. 185, 1-26 (2003)

23. Sjögreen, B., Yee, H.C.: Low dissipative high-order numerical simulations of supersonic reactive flows. Int. J. Numer. Methods Fluids 43, 1221-1238 (2003)

24. Sjögreen, B., Yee, H.C.: A numerical study of resistivity and hall effects for a compressible MHD Model. AIAA Paper No. 50462005, 36th AIAA Plasmadynamics and Lasers Conference, 6-9 June 2005, Toronto, ON, Canada. NAS Technical Report NAS-06005, NASA Ames Research Center (2005)

25. Sjögreen, B., Yee, H.C.: Performance of high order filter methods for a Richtmyer-Meshkov instability. In: Proceedings of the ICCFD4 Conference, 10-14 July 2006, Gent, Belgium (2006)

26. Wheatley, V., Pullin, D.I., Samtaney, R.: Regular shock refraction at an oblique planar density interface in magnetohydrodynamics. J. Fluid Mech. 522, 179-214 (2005)

27. Yee, H.C.: A class of high-resolution explicit and implicit shockcapturing methods. VKI Lecture Series 1989-2004, 6-10 March 1989. NASA TM-101088, Feb. 1989

28. Yee, H.C., Sandham, N.D., Djomehri, M.J.: Low dissipative high order shock-capturing methods using characteristic-based filters. J. Comput. Phys. 150, 199-238 (1999)

29. Yee, H.C., Vinokur, M., Djomehri, M.J.: Entropy splitting and numerical dissipation. J. Comput. Phys. 162, 33-81 (2000)

30. Yee, H.C., Sjögreen, B.: Designing adaptive low dissipative high order schemes for long-time integrations for long-time integrations. In: Drikakis, D., Geurts, B. (eds.) Turbulent Flow Computation. Kluwer, Dordrecht (2002). RIACS Technical Report TR01-28, Dec. 2001

31. Yee, H.C., Sjögreen, B.: Divergence free high order filter methods for the compressible MHD equations. In: Proceedings of the International Conference on High Performance Scientific Computing, 10-14 March 2003, Hanoi, Vietnam (2003)

32. Yee, H.C., Sjögreen, B.: Efficient low dissipative high order scheme for multiscale MHD flows, II: Minimization of Div(B) numerical error. RIACS Technical Report TR03.10, July, 2003, NASA Ames Research Center. J. Sci. Comput. (2005) doi: 10.1007/s10915-005-9004-5, 29, 115-164 (2006)

33. Yee, H.C., Sjögreen, B.: Nonlinear filtering and limiting in high order methods for ideal and non-ideal MHD. In: Proceedings of the ICOSAHOM, 21-25 July 2004, Brown University, RI, J. Sci. Comput. pp. 507-521 (2006)

34. Yee, H.C., Sjögreen, B.: Nonlinear filtering in compact high order schemes. In: Proceedings of the 19th ICNSP \& 7th APPTC Conference, Nara, Japan, 11-15 July 2005, J. Plasma Phys. 72, pp. 833-836 (2006)

35. Yee, H.C., Sjögreen, B.: Development of low dissipative high order filter schemes for multiscale Navier-Stokes/MHD systems. In: Proceedings of the CalSpace/UCR ASTRONUM Conference, Palm Springs, CA, 27-30 March 2006. Expanded version, J. Comput. Phys. 225, 910-934 (2007). http://dx.doi.org/10.1016/ j.jcp.2007.01.012

36. Yee, H.C., Sjögreen, B.: Adaptive filtering and limiting in compact high order methods for multiscale gas dynamics and MHD systems. Special Issue of Computers and Fluids in Honor of Professor Hafez's 6th-birthday (2007) (in press) 